\title{
Streaming potential measurements as a characterization method for nanofiltration membranes
}

\author{
J.M.M. Peeters, M.H.V. Mulder *, H. Strathmann \\ University of Twente, Faculty of Chemical Technology, P.O. Box 217, 7500 AE, Enschede, The Netherlands
}

Received 17 June 1998; accepted 19 October 1998

\begin{abstract}
The streaming potentials of two different nanofiltration membranes were studied with several electrolyte solutions to investigate the influence of salt type and concentration on the zeta potential and kinetic surface charge density of the membranes. The zeta potentials decreased with increasing salt concentration, whereas the kinetic surface charge densities increased. The kinetic surface charge densities could be described by Freundlich isotherms, except in one case, indicating that the membranes had a negligible surface charge. The kinetic surface charge density observed was caused by adsorbed anions. Salt retention measurements showed different mechanisms for salt separation for the two investigated membranes. One membrane showed a salt retention that could be explained by a Donnan exclusion type of separation mechanism, whereas for the other membrane the salt rejection seemed to be a combination of size and Donnan excluion. Comparing the results obtained by the streaming potential measurements with those of the retention measurements, it could be concluded that the membrane with the highest kinetic surface charge density showed the Donnan exclusion type of separation, whereas the membrane with the lower surface charge density showed a separation mechanism that was not totally determined by Donnan exclusion, size effects seemed to play a role as well. (C) 1999 Elsevier Science B.V. All rights reserved.
\end{abstract}

Keywords: Nanofiltration; Salt retention; Streaming potential

\section{Introduction}

The separation mechanism of nanofiltration membranes is assumed to be based on a combination of several processes, like size exclusion, charge exclusion and, as sometimes referred to as, dielectric exclusion [1,2]. In the case of uncharged molecules, mainly size exclusion is thought to be

\footnotetext{
* Corresponding author. Tel.: + 31-53-4892950; fax: + 3153-4894611; e-mail: m.h.v.mulder@ct.utwente.nl.
}

responsible for the separation, whereas concerning the separation of charged species, e.g. ions, both size and charge effects may play a role [3-5].

The charge effects that are partly causing the rejection of ions are resulting from interactions between the charged particles and the charged membrane. These electrochemical interactions between membrane and ions can be investigated in different ways. Separation experiments have been performed, varying parameters like salt concentration and membrane flux, resulting in a value 


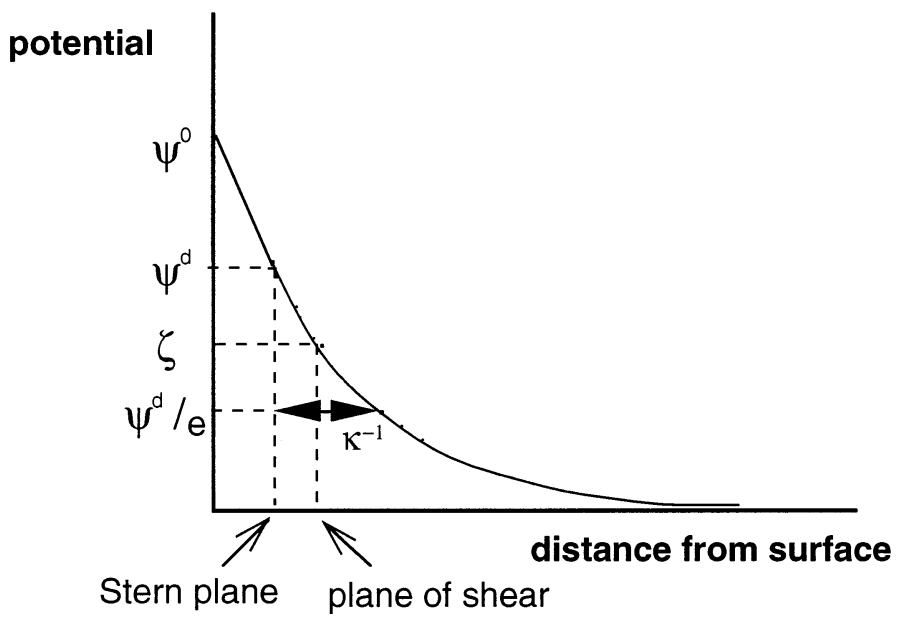

Fig. 1. Schematic drawing of potential decrease as a function of the distance from the surface in an electrolyte solution.

for the membrane charge [6-8]. Membrane potential measurements have been carried out as well to determine the membrane charge density $[6,9,10]$. Furthermore, electrical impedance measurements have been carried out giving an indication for the resistance of electrolyte transport through the membrane [11].

In this paper we will use electrokinetic measurements to investigate the membrane charge of two nanofiltration membranes. Streaming potential measurements have been carried out to determine the zeta potential and the kinetic surface charge density of the membranes. To investigate the influence of the type of the electrolyte and of its concentration, different types of salts at different concentrations were used for the streaming potential measurements. Finally, the results of the streaming potential measurements will be compared to the results of retention measurements carried out with the same electrolyte solutions as used in the retention measurements.

\section{Theory}

\subsection{Streaming potential}

Ions in an electrolyte solution that is brought in contact with a charged surface will not be distributed randomly. The concentration of counter- ions near the membrane will be higher than that in the bulk of the solution. The charges at and adjacent to the surface will cause a potential difference between the region near the surface and the bulk of the solution. The potential decreases within the solution as a function of the distance from the charged surface, as shown in Fig. 1. Three different potentials are shown in this figure, the surface potential, $\psi^{0}$, the potential at the Stern plane, $\psi^{\mathrm{d}}$, and the electrokinetic or zeta potential, $\zeta$. All these potentials are defined with respect to the potential at infinite distance from the surface. Although the surface potential is an important parameter, the potential at the Stern plane is practically of more importance. This Stern plane is the interface between the fixed part, i.e. a layer of immobile ions near the charged surface, and the diffusive (mobile) part of the electrical double layer. The potential at the Stern plane is the actual potential influencing the behavior of the charged species. However, as this potential cannot be measured directly, the electrokinetic potential is often considered as an adequate substitute. The plane at which the zeta potential is located should be outside the Stern layer, and represents the potential at the surface of shear between surface and solution where there is relative motion between them. The position of the surface of shear is close to, and from practical reasons assumed to be identical to, the Stern 
surface [12]. The zeta potential is shown in Fig. 1 as well. The zeta potential can be determined by electrokinetic measurements, like streaming potential and electro-osmosis measurements.

A streaming potential is the potential difference at zero current caused by the convective flow of charge due to a pressure gradient through a charged capillary, plug, diaphragm or membrane. A streaming potential is generated by exerting a force on the double layer that has been built up in the solution near the charged surface. Since an excess of counter charges is present, movement of those counter charges causes a current. This current which is streaming through the double layer is called the streaming current. The accumulation of counter charges downstream generates a streaming potential across the capillary which on its turn causes a conduction current through the capillary in the reverse direction. In steady state, the streaming current equals the conduction current.

Measurement of a streaming potential can provide both the zeta potential, $\zeta$, and the net charge density, $\sigma^{\mathrm{d}}$, at the hydrodynamic shear plane. The streaming potential is defined to be positive if the higher potential is at the high pressure side.

The relation between the zeta potential, $\zeta$, the streaming potential, $\Delta E_{\mathrm{str}}$, and the pressure difference applied, $\Delta P$, is given by $[12,13]$ :

$\Delta E_{\mathrm{str}}=\frac{\varepsilon \zeta \Delta P}{\eta\left(\lambda_{0}+\frac{2 \lambda_{\mathrm{s}}}{r}\right)}$

where $\varepsilon$ is the permittivity of the medium, $\eta$ the viscosity, $\lambda_{0}$ the bulk conductivity, $\lambda_{\mathrm{s}}$ the surface conductivity, and $r$ the radius of the pore or capillary or half the width of a slit.

It is difficult to measure directly the surface conductivity and therefore, at low electrolyte concentrations, where the surface conductivity is not be negligible, Eq. (1) cannot easily be applied. This problem can be circumvented by measuring the actual resistance of the electrolyte solution across the slit or pore, $R_{\text {exp }}$, and comparing this value with the resistance that can be calculated from experiments at high concentrations, where the surface conductivity can be neglected, $R_{\mathrm{th}}$ [13]. Then, Eq. (1) can be written as:
$\Delta E_{\mathrm{str}}=\frac{\varepsilon \zeta \Delta P}{\eta \lambda_{0}} \frac{R_{\mathrm{exp}}}{R_{\mathrm{th}}}$

In the latter case the streaming potential is not dependent on the geometry of the capillary.

In the case of a low potential, the relation between the surface charge density at the hydrodynamic shear plane and the zeta potential is given in the following equation:

$\sigma^{d}=\frac{\varepsilon \zeta}{\kappa^{-1}}$

where $\kappa^{-1}$ is the Debije length. The Debije length can be calculated by Eq. (4):

$\kappa^{-1}=\sqrt{\frac{\varepsilon k T}{4 e^{2} N_{\mathrm{A}} I}}$

with $I=0.5 \Sigma z_{\mathrm{i}}^{2} c_{\mathrm{i}}$ : where $\varepsilon$ is the permittivity of the medium, $k$ the Boltzmann constant, $T$ the temperature, $e$ the elementary charge, $N_{\mathrm{A}}$ the Avogadro number, $I$ the ionic strength, $z_{\mathrm{i}}$ the valency and $c_{i}$ the concentration of species $i$. Although Eq. (4) was derived for symmetrical electrolytes, like 1-1 and 2-2 electrolytes, we will use it for 1-2 and 2-1 electrolytes as well.

Concerning the membrane charge density, a distinction can be made between the actual charge of the membrane, which originates from membrane fixed charges, like carboxylic or sulphonic groups, and adsorbed charges.

Benavente et al. [14] introduced a method to discriminate between these two types of charges. Therefore, it was assumed that three components added to the total charge of the system. First, the fixed charges at the membrane surface, $\Sigma^{0}$, second, the charges of the Stern-layer, $\Sigma^{\mathrm{s}}$, and finally, the charges within the diffusive part of the electric double layer, $\Sigma^{\mathrm{d}}$. The charge of the Stern-layer is caused by the adsorption of ions. As electroneutrality is assumed, the total charge equals zero.

$\sum^{0}+\sum^{\mathrm{s}}+\sum^{\mathrm{d}}=0$

Assuming that the diameter of the pore or the width of the slit through which the solution is streamed is much larger than the Stern-layer thickness, the relation between the charge densities, $\sigma$, of each of the layer can be written as: 
$\sigma^{0}+\sigma^{\mathrm{s}}+\sigma^{\mathrm{d}}=0$

because all charges are spread over an equal area.

Both co- and counter-ions can adsorb at charged membrane surfaces due to electrostatic and non-electrostatic interactions. As anions in the vicinity of non-polar surfaces are less hydrated than cations, they can adsorb more closely to the surface, resulting often in an excess of negative charges in the layer nearest to the surface and, therefore, in an apparent negative surface charge $[15,16]$.

The total molar Gibbs free energy of adsorption is the sum of an electrostatic and a chemical (specific) part, $\Delta G_{\mathrm{c}}$ :

$\frac{\Delta G_{\mathrm{ads}}}{R T}=\frac{z_{-} \zeta}{z_{+}}+\frac{\Delta G_{\mathrm{c}}}{R T}$

The charge of the Stern-layer is caused by the adsorbed ions. One of the adsorption types by which the total amount of charge is related to the concentration of ions is the Freundlich adsorption isotherm, describing adsorption on non-uniform sites. This empirical isotherm describes the relation between between the charge density and the fraction of anions by a power law [14]:

$\sigma^{s}\left(x_{-}\right)=a x_{-}^{b}$

with $a$ and $b$ being constants, and

$x_{-}=\frac{c v_{-} M_{\mathrm{H}_{2} \mathrm{O}}}{\rho-c\left[v_{+} M_{+}+v_{-} M_{-}-\left(v_{+}-v_{-}\right) M_{\mathrm{H}_{2} \mathrm{O}}\right]}$

the fraction of anions in solution. In this equation $c$ is the electrolyte concentration, $v$ the stoichiometric number, $M$ the molecular weight, and $\rho$ the density, while the subscripts,,-+ , and $\mathrm{H}_{2} \mathrm{O}$ refer to the anion, the cation and water, respectively.

The total amount of charge within the membrane, adsorbed layer and diffusive layer is then:

$\sigma^{d}=\sigma^{0}+a x_{-}^{b}$

From this equation the number of adsorption sites, $N$, and the free Gibbs energy of adsorption $\left\langle G_{\text {ads }}\right\rangle$ can be determined according to [14]:

$N=\frac{a}{z_{-} e} \frac{\sin \pi b}{\pi b}$ and

$$
\left\langle\Delta G_{\mathrm{ads}}\right\rangle=-\frac{R T}{b}
$$

\subsection{Donnan exclusion}

Electrostatic interaction between ions and charges at the membrane surface is one of the mechanisms of nanofiltration membranes retaining ions. The repulsion of the ions from the surface can be described by Donnan exclusion.

If a charged membrane is put in contact with an ionic solution, ions with the same charge sign as that of the membrane (the co-ions) are excluded and cannot pass the membrane, whereas the ions with the opposite charge sign as that of the membrane (counter-ions) are able to pass the membrane in principle [17].

Because of the charge of the membrane, a concentration difference of the ions between the solution and the membrane is built up, leading to an osmotic pressure difference between the membrane and the solution. As thermodynamic equilibrium is assumed, an additional potential across the membrane, the Donnan potential, $E_{\text {Don }}$, will compensate this osmotic pressure difference. For ideal solutions, the Donnan potential is given by:

$E_{\text {Don }}=\frac{R T}{z_{\mathrm{i}} F} \ln \frac{c_{\mathrm{i}, \mathrm{m}}}{c_{\mathrm{i}}}$

where $R, T, z$ and $F$ having their usual meaning and with $c_{\mathrm{i}}$ and $c_{\mathrm{i}, \mathrm{m}}$ the concentration of species $\mathrm{i}$ in the bulk and the membrane, respectively.Combining the requirements of identical chemical potentials in membrane and bulk and of electroneutrality, for a 1-1 electrolyte the relation between concentration of the co-ion in the bulk, its concentration in the membrane and the fixed charges at the membrane surface, $c_{\mathrm{x}, \mathrm{m}}$, can be written as [15]:

$c_{\mathrm{co}-\text { ion }, \mathrm{m}} \times c_{\mathrm{x}, \mathrm{m}}+\left(c_{\mathrm{co}-\text { ion }, \mathrm{m}}\right)^{2}=\left(c_{\mathrm{co}-\text { ion }}\right)^{2}$

The Donnan potential is dependent on several factors, e.g.:

* salt concentration;

* fixed charge concentration in the membrane;

* valence of the co-ion; 


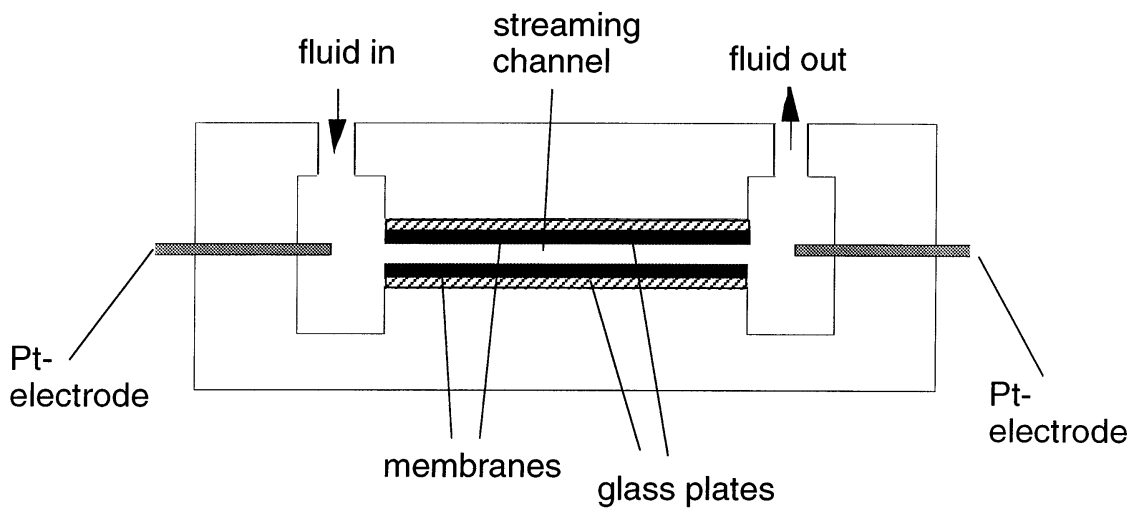

Fig. 2. Schematic illustration of the streaming potential system.

* valence of the counter-ion.

With increasing salt concentration (and increasing ionic strength) and decreasing fixed membrane charge, the concentration of the co-ion in the membrane increases, as shown in Eq. (13). This decrease of co-ion exclusion from the membrane leads to a lower rejection of the salt, because the rejection of the co-ion determines the rejection of the salt.

A higher valence of the co-ion and a lower valence of the counter-ion will cause an increase of the Donnan potential and, therefore, an increase in retention.

\section{Experimental}

\subsection{Streaming potential measurements}

The set-up to determine streaming potentials was almost identical to that described by Van Wagenen and Andrade [18]. With this kind of set-up, streaming potentials can be measured along a surface, rather than through a surface. In literature streaming potential measurements through membranes or other porous species are described as well [19-23]. In those papers, mainly the features of the pores are then determined.

Fig. 2 shows a schematic drawing of the cell which is used to support the membrane samples for the streaming potential measurements. The cell was manufactured by the Agricultural Univer- sity of Wageningen (the Netherlands). The membrane samples are glued upon glass plates (microscope slides). The dimensions of the membrane samples are $76 \times 26 \mathrm{~mm}$.

The cell consists of two plexiglass parts containing sample channels that hold the glass slides with the membranes glued on top. Clamps hold both cell parts together. Under the glass slides silicon rubber sheets can be put to prevent leakage at the interface between cell and glass slide. The cell parts are separated by a $200 \mu \mathrm{m}$ thick Teflon sheet which serves two functions: spacing between sample plates and leak prevention. Platina black electrodes are inserted into the chambers at both ends of the cell.

The driving pressure can be applied in either direction and consequently the electrolyte solution can pass through the channel. The pressure was varied in the range of 0 to 0.25 bar and was monitored with an accuracy of 0.01 bar. Streaming potential measurements were repeated at least six times by measuring at decreasing pressure.

The streaming potential was measured using a digital multimeter (Simpson, Model 464, Simpson Electric, Elgin), which had an internal impedance of $10 \mathrm{G} \Omega$.

The electrolyte solutions used were in the range of $0.01-10^{-5} \mathrm{M}$ for the three salts used: $\mathrm{NaCl}$, $\mathrm{CaCl}_{2}$, and $\mathrm{Na}_{2} \mathrm{SO}_{4}$ (Merck, PA).The streaming potentials were measured from low to high concentration. For every salt, fresh membranes were used. 


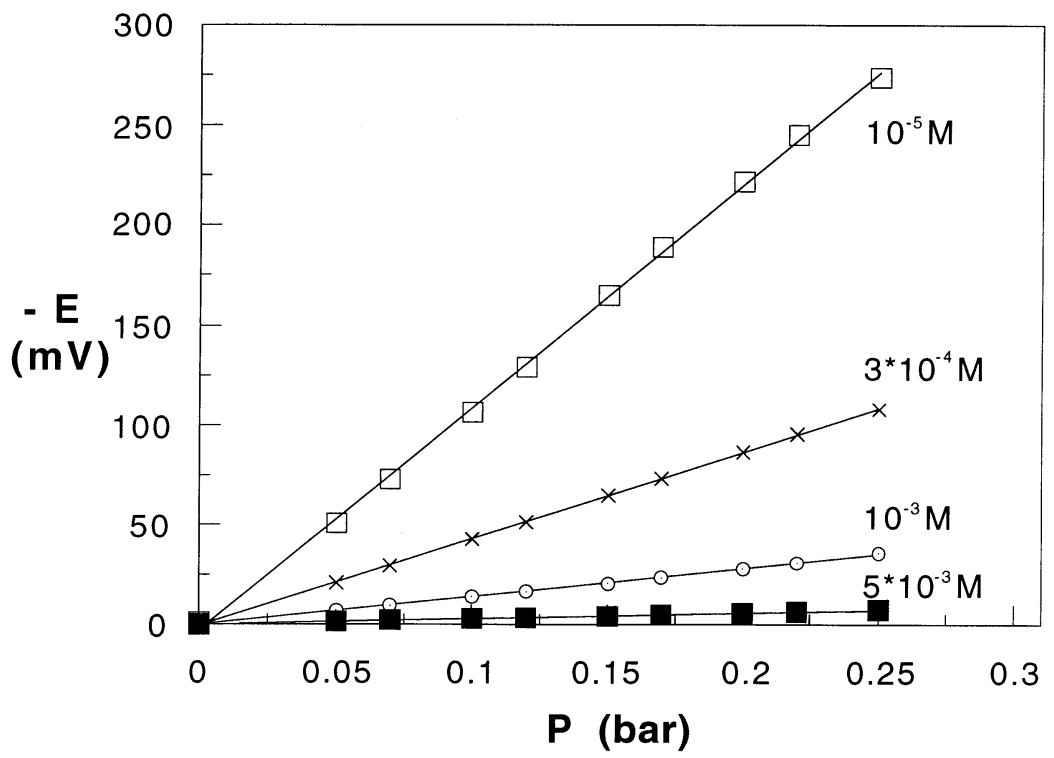

Fig. 3. Streaming potential as a function of the applied pressure for different concentrations of NaCl. Membrane: NF45.

Electrolyte conductance was measured by a conductivity meter (Microprocessor Conductivity Meter LF 537, WTW). The $\mathrm{pH}$ of the electrolyte bulk solutions was measured by a $\mathrm{pH}$ meter (691 pH Meter, MetrOhm). Demineralized water filtered by a Milli-Q-Plus unit (conductivity $60 \mathrm{nS}$ $\mathrm{cm}^{-1}$ ) was used as solvent. The temperature of the system was $20-23^{\circ} \mathrm{C}$. The membranes used were two commercially available nanofiltration membranes: ASP35 (Advanced Membrane Technology) and NF45 (Dow-FilmTec).

\subsection{Retention measurements}

Retention measurements were carried out with $\mathrm{CaCl}_{2}, \mathrm{NaCl}$, and $\mathrm{Na}_{2} \mathrm{SO}_{4}$ (Merck, PA), respectively, at three different initial concentrations, $0.001,0.005$, and $0.01 \mathrm{M}$. The solvent was demineralized water filtered by a Milli-Q-Plus unit. The retention measurements were carried out in a stirred dead-end filtration set-up at a pressure of 5 bar. The flux through the membrane was automatically recorded.

First, all membranes were permeated with water for $4 \mathrm{~h}$. Then, the feed solution was changed from pure water to a salt solution. After a stabilization time of $2 \mathrm{~h}$ the concentration of the feed was measured and subsequently permeation samples were taken. Salt retention measurements were started with the lowest concentration. The sequence of the salts used was $\mathrm{CaCl}_{2}, \mathrm{NaCl}$, and $\mathrm{Na}_{2} \mathrm{SO}_{4}$, respectively. In between retention measurements with different salts, the membrane was flushed for at least $2 \mathrm{~h}$ with pure water.

\section{Results and discussion}

\subsection{Streaming potential measurements}

The streaming potentials of both membranes decreased while increasing the concentration of the electrolyte concentration for all types of electrolytes. In Fig. 3 this is illustrated using $\mathrm{NaCl}$ as electrolyte solution for the NF45 membrane.

The use of different electrolytes of identical ionic strength resulted in different streaming potentials and, therefore, in different zeta-potentials. The Figs. 4 and 5 show the zeta potentials for both membranes, as calculated from the stream- 


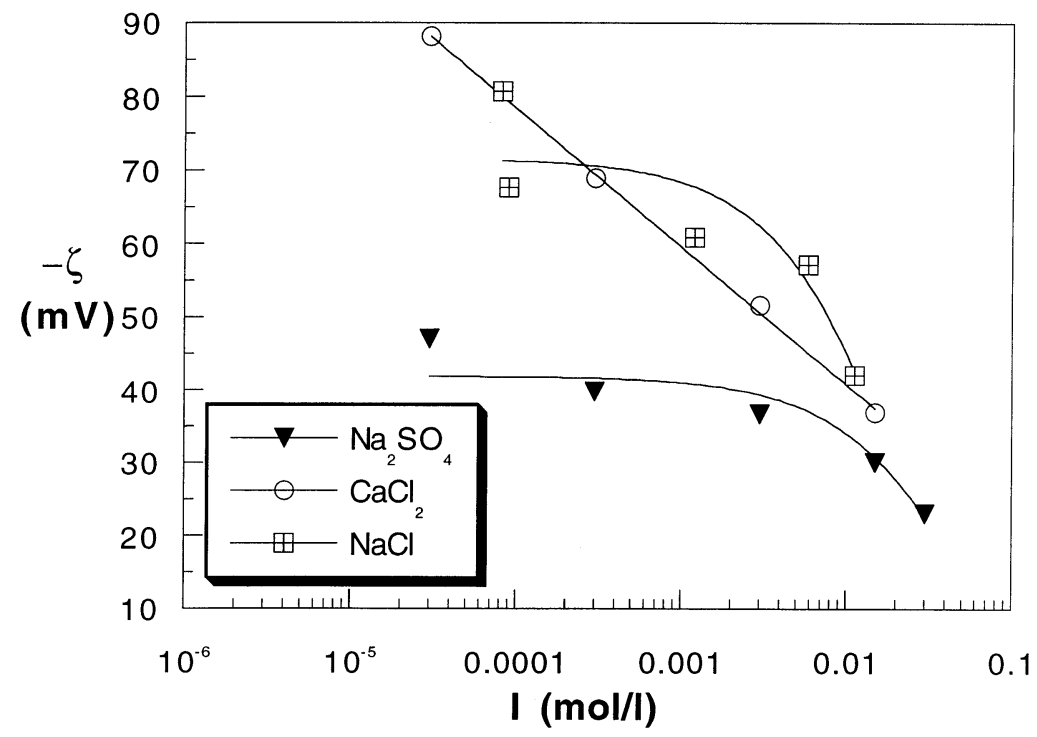

Fig. 4. Zeta-potentials for a ASP35 membrane measured with different electrolyte solutions at various concentrations.

ing potentials according to Eq. (2). For the ASP35 membrane, the zeta potentials determined for the sulphate solution were lower than those for the sodium and calcium chloride solutions. The values for both chloride solutions are comparable. For the NF45 membrane, the highest zeta-potentials were determined with the $\mathrm{Na}_{2} \mathrm{SO}_{4}$ solutions. The zeta-potentials determined with $\mathrm{NaCl}$ were lower, whereas those for $\mathrm{CaCl}_{2}$ were the lowest. Especially in the case of the lower electrolyte concentrations, the ASP35 membrane showed much higher zeta potentials for the chloride solutions compared to the NF45 membrane.

The kinetic surface charge densities calculated from the streaming potential measurements by Eq. (3) are shown in Figs. 6 and 7. In these figures the kinetic surface charge densities of NF45 and ASP35 membranes are shown as functions of the anion fractions in the $\mathrm{NaCl}$ and the $\mathrm{CaCl}_{2}$ solutions to discriminate between the actual charge of the membrane and the adsorbed charges. The dashed lines within these figures represent Eq. (9), assuming adsorption according to the Freundlich isotherm. The solid lines within the figures represent the equation: $\sigma^{d}=a x^{b}$

where the actual charge density of the membrane is neglected and the charge density at the Stern-layer equals the charge density at the shear plane. Using a log-log plot with the parameters $\sigma^{\mathrm{d}}$ and $x_{-}$at the axes, a straight line should result with a slope equal to the parameter $b$, which is indeed the case. As can be seen from both figures the differences between the two lines according to Eqs. (9) and (14) are very small, indicating that the actual charge densities of the two membranes were small as well. The charge densities at the membrane surface calculated by Eq. (9) were in the range of $-4 \times$ $10^{-5}-2 \times 10^{-4} \mathrm{C} \mathrm{m}^{-2}$, which are indeed small compared to the values of the calculated total charge density as can be seen from the Figs. 6 and 7 .

Comparing the ASP35 and the NF45 membrane, the kinetic surface charge densities of the ASP35 membrane calculated from the streaming potential measurements with the chloride solutions are higher than those of the NF45 membrane. From Table 1 it can be seen that especially the amounts of adsorption sites, calcu- 


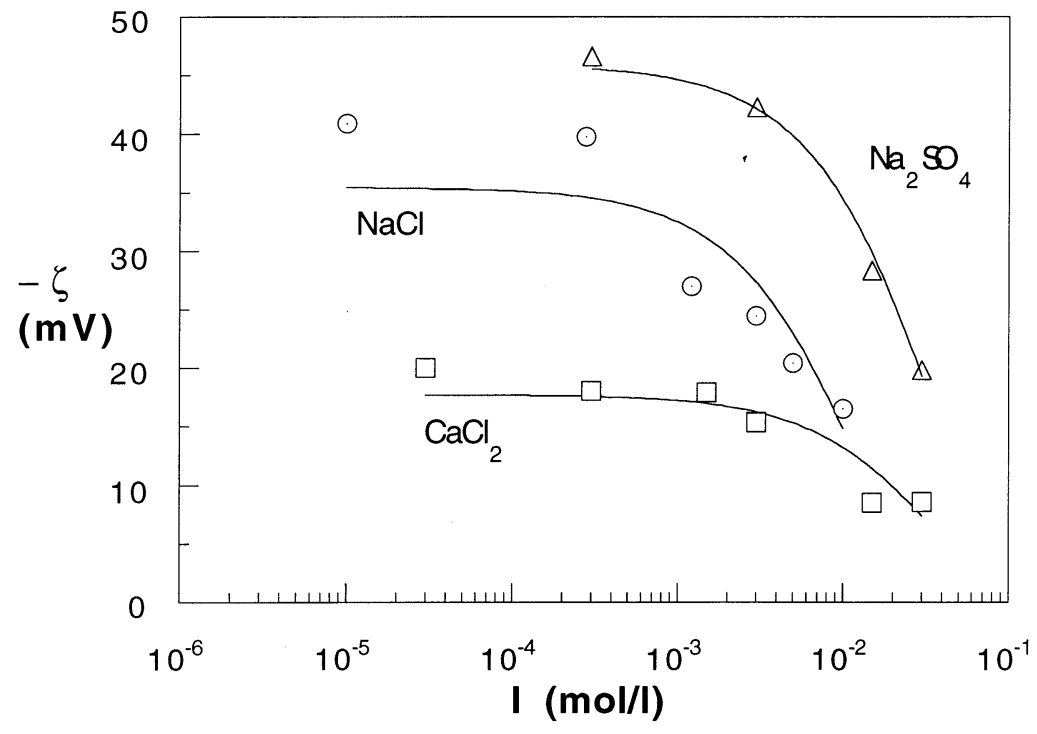

Fig. 5. Zeta-potentials for a NF45 membrane measured with different electrolyte solutions at various concentrations.

lated by Eq. (10), differed for both membranes, while the adsorption free energies were all in the same order of magnitude.

In the case of the ASP35 membrane the fitting of the kinetic surface charge density versus the anion fraction resulted in comparable values for the amounts of adsorption sites and only slight differences between the free energies of adsorption for both chloride solutions. The calculated parameters $N$ and $\left\langle G_{\text {ads }}\right\rangle$ obtained for the $\mathrm{NaCl}$ and $\mathrm{CaCl}_{2}$ solution in the case of the NF45 membrane, were comparable as well, although in this case the free energies of adsorption were identical, whereas the calculated amounts of adsorption sites showed small differences. For the former membrane, the calculated amount of adsorption sites was different for the sulphate ions compared to that of the chloride ions. For both membranes it seems to be reasonable to assume that the main source for charge at the membrane surface was due to anion adsorption, whereas cation adsorption was negligible. The same kind of results was observed for a polysulfone membrane by Benavente et al. [14] and for polyamide membranes by Hernandez-Gimenez et al. [24].
The results of the data fitting using the Freundlich isotherm are shown in Table 1.

In the case of the NF45 membrane, the fitting of the $\mathrm{Na}_{2} \mathrm{SO}_{4}$ streaming potential data with the Freundlich isotherm gave no reliable results.

\subsection{Retention measurements}

Retention measurements carried out with the NF45 and ASP35 membranes showed a different behaviour for both. For both membranes the retention for $\mathrm{Na}_{2} \mathrm{SO}_{4}$ is the highest compared to that of the other salts (see Figs. 8 and 9), but in the case of the ASP35 membrane the retention of $\mathrm{NaCl}$ is higher than that of $\mathrm{CaCl}_{2}$, whereas for the NF45 membrane it is inversed.

Most probably, the differences in rejection characteristics between the two membranes are caused by the differences in the determining separation mechanisms. In the case of the ASP35 membrane the retention results can be interpreted by the Donnan exclusion mechanism, based on interactions between the charges of the membrane and those of the ions. The retention experiments for the NF45 membrane cannot be explained exclusively by Donnan exclusion, and 


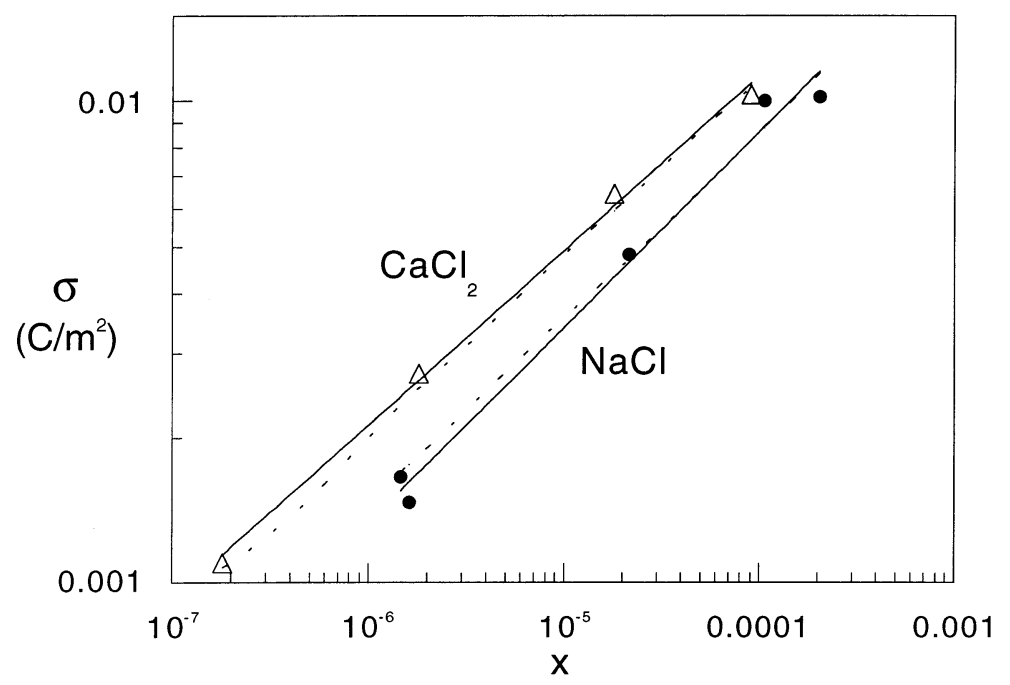

Fig. 6. Charge density of the ASP35 membrane as a function of the anion fraction with $\mathrm{NaCl}$ and $\mathrm{CaCl}_{2}$ solutions.

most probably size effects play a role for salt separation with this membrane as well.

The ASP 35 membrane shows a retention sequence of $R\left(\mathrm{Na}_{2} \mathrm{SO}_{4}\right)>R(\mathrm{NaCl})>R\left(\mathrm{CaCl}_{2}\right)$, so the retention for the bivalent anion is the largest, whereas that of the bivalent cation is the smallest. The retention of the $1-1$ salt $(\mathrm{NaCl})$ is in between the other two. If it is assumed now, that the ASP35 membrane is negatively charged, the high retention for the sodium sulphate (bivalent co-ion, monovalent counter-ion) and the low(er) retention for calcium chloride (bivalent counter-ion, monovalent co-ion) fit well with the Donnan exclusion model. The decrease of the retention with increasing concentration can also be explained by assuming a decreasing influence of Donnan exclusion.

The results obtained for the NF45 membrane may not be explained by Donnan exclusion, because both the retention for the bivalent cation and that of the bivalent anion are high. Neither can the retention sequence exclusively be explained by differences in the size of the different ions. Although the sizes of a hydrated sulphate and calcium ion are larger than those of sodium and chloride, the calcium ion is in turn larger than the sulphate [25]. So, in the case of separa- tion based on size exclusion only, calcium would have shown the highest retention. Possibly, the retention behavior of the NF45 membrane is determined by a combination of both charge and size exclusion.

\subsection{Comparison between streaming potential and retention measurements}

Comparing the results obtained from the streaming potential measurements with sodium and calcium chloride for the NF45 and the ASP35 membrane, it can be concluded that the actual surface charge for both membranes is only small. However, the adsorbed charge differs largely when comparing the two membranes and is much higher in the case of the ASP35 membrane. The difference in adsorbed charge may be the reason for the discrepancy between the ion separation characteristics of both membranes. The adsorbed charge at the ASP35 membrane is so large that the membrane behaves in the retention measurements like a negatively charged membrane. As the charge densities for both the sodium and calcium chloride solutions are comparable, the difference in retention seems to be caused by the difference in 


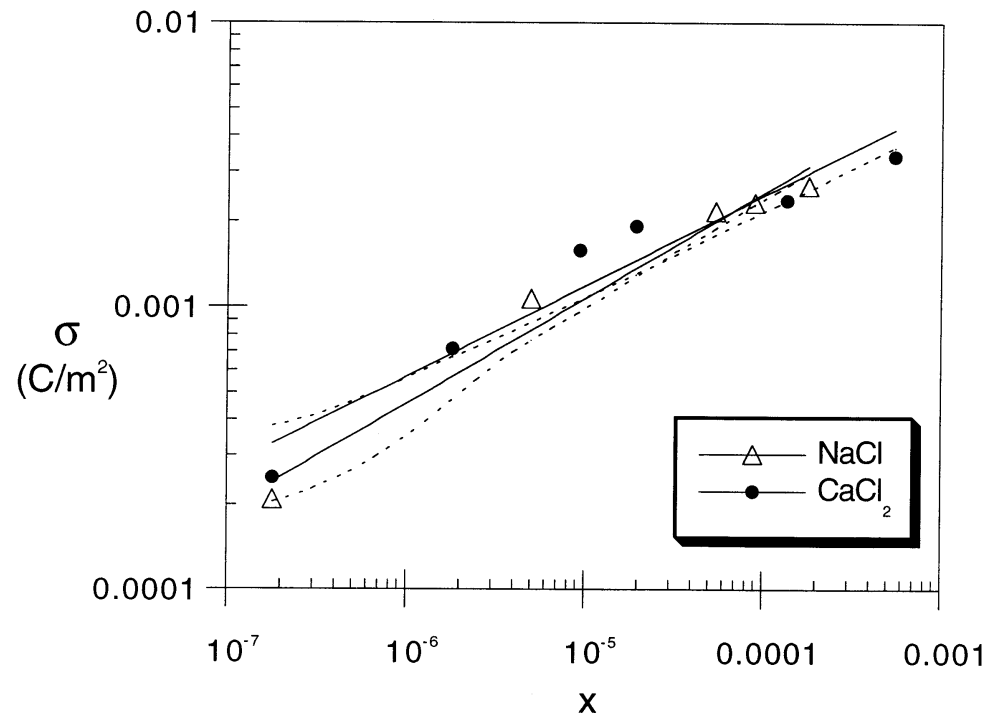

Fig. 7. Charge density of the NF45 membrane as a function of the anion fraction determined with $\mathrm{NaCl}$ and $\mathrm{CaCl}{ }_{2}$ solutions.

valency of the counter-ions for these two salts. Because the Donnan-exclusion becomes smaller if the valency of the counter-ion becomes higher, this can explain the higher retention for the sodium chloride solution.

With a sodium sulphate solution, the charge density of the ASP35 membrane is somewhat smaller than with sodium and calcium chloride solutions, although the retention of the sodium sulphate solution is higher than that of both chloride solutions. Most probably, this higher retention is caused by an increase in repulsion due to the valency of the co-ion changing from mono- to bi-valent.

In the case of the NF45 membrane, the adsorbed charge is not large enough to determine the salt separation completely. The retention for calcium chloride is higher than that of sodium chloride, which is most probably caused by a difference in size between the calcium and sodium ion. In the case of a sodium sulphate solution the charge density is highest, resulting in the highest retention for this salt as well. This may be caused by a combination of larger electrostatic repulsion in the case of the sulphate ion and by size exclusion.

\section{Conclusions}

Both streaming and zeta potentials decreased with increasing salt concentration. The zeta potentials and the calculated charge densities are higher for the ASP35 membrane than for the NF45 membrane. The actual charge density of both membranes is small and especially at higher electrolyte concentrations, it is negligible compared to the adsorbed charges. Anion adsorption seems to be the main source for the apparent membrane charge, whereas cation adsorption is small. In most cases anion adsorption could be described with a Freundlich adsorption isotherm, only sodium sulphate adsorption on the NF45 membrane could not be described by this type of isotherm.

Retention measurements showed a different salt separation behaviour for the ASP35 and the NF45 membrane. For the ASP35 membrane the differences in retention between sodium sulphate, sodium chloride and calcium chloride seemed to be caused by Donnan exclusion, whereas for the NF45 size effects seems to play a role as well, especially when the retentions for calcium and sodium chloride are compared. Most probably, the ASP35 membrane shows 
Table 1

Parameters $a$ and $b$, according to Eq. (15), the number of adsorption sites, $N$, and the free Gibbs energy of adsorption, according to Eqs. (11) and (12), respectively, for the two nanofiltration membranes

\begin{tabular}{llccc}
\hline Membrane: ASP35 & $a\left(\mathrm{C} \mathrm{m}^{-2}\right)$ & $b(-)$ & $N(-)$ & $\left\langle\Delta G_{\mathrm{ads}}\right\rangle\left(\mathrm{kJ} \mathrm{mol}^{-1}\right)$ \\
\hline $\mathrm{NaCl}$ & 0.36 & 0.41 & $1.7 \times 10^{18}$ & -6.1 \\
$\mathrm{CaCl}_{2}$ & 0.32 & 0.36 & $1.6 \times 10^{18}$ & -6.8 \\
$\mathrm{Na}_{2} \mathrm{SO}_{4}$ & 0.27 & 0.41 & $6.3 \times 10^{17}$ & -6.0 \\
$\mathrm{Membrane}:$ NF45 & & & & -6.7 \\
$\mathrm{NaCl}$ & 0.07 & 60.37 & $3.8 \times 10^{17}$ & -6.7 \\
$\mathrm{CaCl}_{2}$ & 0.08 & 80.37 & $4.4 \times 10^{17}$ & \\
\end{tabular}

this Donnan type of retention behavior because of its high kinetic surface charge. The adsorbed charge at the NF45 membrane is not high enough to discriminate on the basis of charge between sodium and calcium chloride. Therefore, size exclusion seems to determine the difference in separation between these two salts.

\section{Acknowledgements}

The authors would like to thank the European Union (Science Programme) for the financial support for this project. Furthermore, the authors would like to thank Maikel van Bree, Jeroen van Beckum, and Edwin van der Steeg for their experimental work.

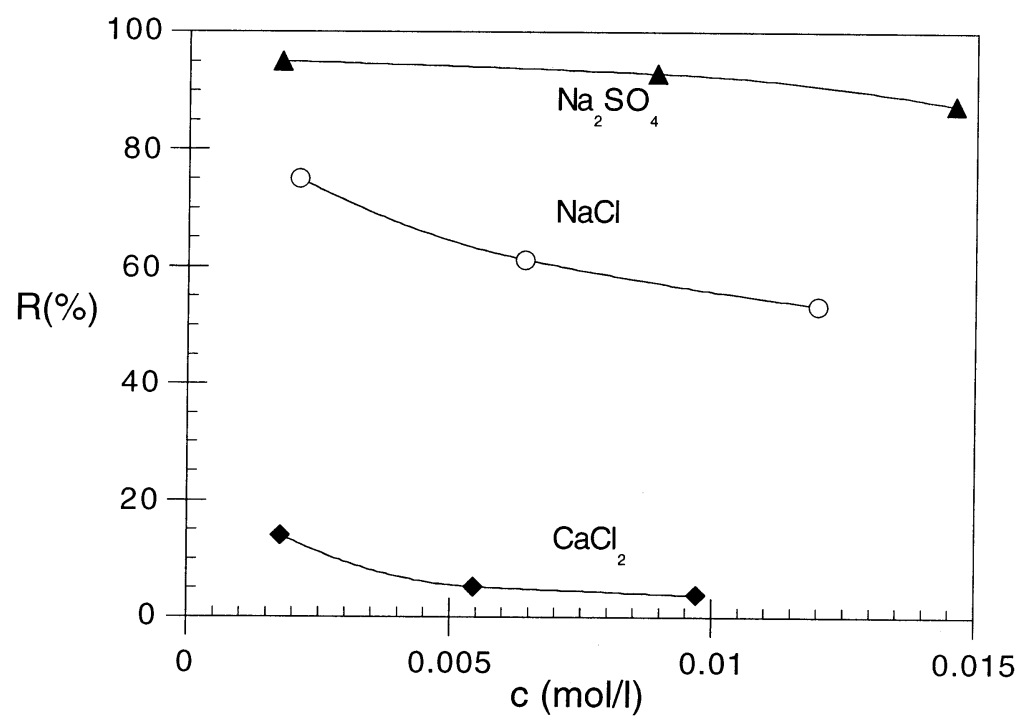

Fig. 8. Retention vs. concentration of various salts for a ASP35 membrane. Pressure: 5 bar. 


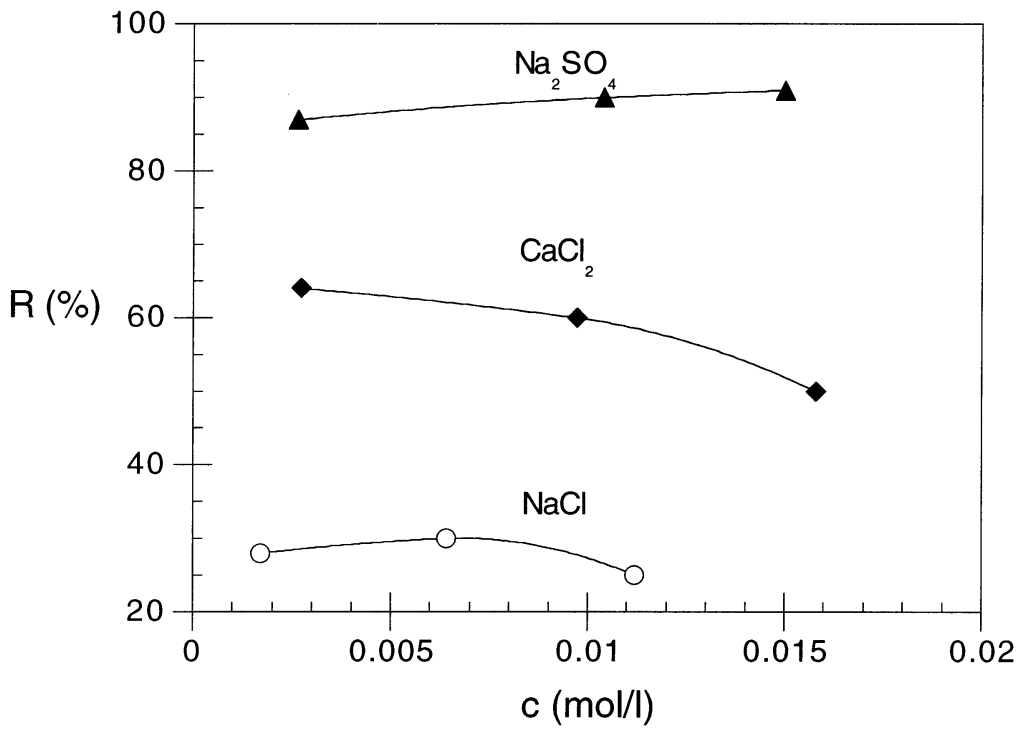

Fig. 9. Retention vs. concentration of various salts for a NF45 membrane. Pressure: 5 bar.

\section{References}

[1] A. Yaroshchuk, E. Staude, Charged membranes for low pressure reverse osmosis properties and applications, Deslination 79 (1992) 1-15.

[2] A.G. Fane, A.R. Awang, M. Bolko, et al., Metal recovery from waste water using membranes, Water Sci. Technol. 25 (1992) 5.

[3] S. Alami-Younssi, A. Larbot, M. Persin, J. Sarrazin, L. Cot, Rejection of mineral salts on a gamma alumina nanofiltration membrane: application to environmental process, J. Membr. Sci. 102 (1995) 123.

[4] T. Tsuru, X.L. Wang, S.I. Nakao, S. Kimura, Transport of neutral and charged solutes through nanofiltration membranes, in: The International Symposium on Fiber Science and Technology, Yokohama, Japan, 1994.

[5] X.L. Wang, T. Tsuru, M. Togoh, S.I. Nakao, S. Kimura, Transport of organic electrolytes with electrostatic and steric-hindrance effects through nanofiltration membranes, J. Chem. Eng. Jpn. 28 (1995) 372.

[6] T. Tsuru, S.I. Nakao, S. Kimura, Effective charge density and pore structure of charged ultrafiltration membranes, J. Chem. Eng. Jpn. 23 (1990) 604.

[7] I. Jitsuhara, S. Kimura, Rejection of inorganic salts by charged ultrafiltration membranes made of sulfonated polysulfone, J. Chem. Eng. Jpn. 16 (1983) 394.

[8] X.L. Wang, T. Tsuru, M. Togoh, S.I. Nakao, S. Kimura, Evaluation of pore structure and electrical properties of nanofiltration membranes, J. Chem. Eng. Jpn. 28 (1995) 186.

[9] Y. Kimura, H.J. Lim, T. Iijima, Membrane potentials of charged cellulosic membranes, J. Membr. Sci. 18 (1984) 285.

[10] R. Takagi, M. Nakagaki, Membrane charge of microporous glass membrane determined by the membrane potential method and its pore size dependency, J. Membr. Sci. 111 (1996) 19.

[11] J. Benavente, G. Jonsson, Transport of $\mathrm{Na}_{2} \mathrm{SO}_{4}$ and $\mathrm{MgSO}_{4}$ solutions through a composite membrane, J. Membr. Sci. 80 (1993) 275.

[12] H. Lyklema, Fundamentals of Interface and Colloid Science: Part II Solid-Fluid Interfaces, Academic Press, London, 1996.

[13] R.J. Hunter, Zeta Potential in Colloid Science, Academic Press, London, 1981.

[14] J. Benavente, A. Hernández, G. Jonsson, Proper and adsorbed charges on the surfaces of the polysulfonic support of a composite membrane from electrokinetic phenomena, J. Membr. Sci. 38 (1993) 211.

[15] C. Werner, H.J. Jacobasch, G. Reichelt, Surface charachterization of hemodialysis membranes based on streaming potential measurements, J. Biomater. Sci. Polym. Ed. 7 (1995) 61.

[16] M. Elimelech, W.H. Chen, J.J. Waypa, Measuring the zeta (electrokinetic) potential of reverse osmosis membranes by a streaming potential analyzer, Desalination 95 (1994) 269.

[17] M. Mulder, Basic Principles of Membrane Technology, Kluwer Academic, Dordrecht, 1996.

[18] R.A. Van Wagenen, J.D. Andrade, Flat plate streaming potential investigations: hydrodynamics and electrokinetic theory, J. Colloid Interface Sci. 76 (1980) 305. 
[19] M. Nyström, M. Lindström, E. Matthiasson, Streaming potential as a tool in the characterization of ultrafiltration membranes, Colloids Surf. 36 (1989) 297.

[20] M. Nyström, A. Pihlajamäki, N. Ehsani, Characterization of ultrafiltration membranes by simultaneous streaming potential measurements and flux measurements, J. Membr. Sci. 87 (1994) 245.

[21] A. Hernández, F. Martínez, A. Martin, P. Prádanos, Porous structure and surface charge density on the walls of microporous alumina membranes, J. Colloid Interface Sci. 173 (1995) 284.

[22] C. Causserand, M. Nystöm, P. Aimar, Study of stream- ing potentials of clean and fouled ultrafiltration membranes, J. Membr. Sci. 88 (1994) 211.

[23] N. Kaneko, Y. Yamamoto, Membrane potential in reverse osmosis, J. Chem. Eng. Jpn. 9 (1976) 158.

[24] A. Hernández-Gimenez, L. Martínez, M.V. Gomez, Concentration dependence of the adsorbed charges and the zeta potentials on the walls of neutral and charged microporous membranes, J. Colloid Interface Sci. 158 (1993) 429.

[25] A.L. Horváth, Handbook of Aqueous Electrolyte Solutions, Ellis Horwood, Chichester, 1985. 